\title{
Electronic structure of indium selenide probed by magnetoabsorption spectroscopy under high pressure
}

\author{
Marius Millot, ${ }^{1, *}$ Jean-Marc Broto, ${ }^{1}$ Sylvie George, ${ }^{1}$ Jesús González, ${ }^{2,3}$ and Alfredo Segura ${ }^{4}$ \\ ${ }^{1}$ Laboratoire National des Champs Magnétiques Intenses (LNCMI), CNRS UPR 3228, Université de Toulouse, 143 Avenue de Rangueil, \\ 31400 Toulouse, France \\ ${ }^{2}$ Malta Consolider Team, DCITIMAC, Universidad de Cantabria, Avenida Los Castros S/N, 39005 Santander, Cantabria, Spain \\ ${ }^{3}$ CES, Universidad de Los Andes, Mérida, Venezuela \\ ${ }^{4}$ MALTA Consolider Team, ICMUV, Universidad de Valencia, c/Dr. Moliner 50, 46100 Burjassot, Valencia, Spain
}

(Received 5 April 2010; published 24 May 2010)

\begin{abstract}
We report on an investigation of the peculiar electronic structure of the layered semiconductor InSe by magneto-optical experiments under high pressure up to $5 \mathrm{GPa}$. Magneto-absorption spectroscopy is performed under pulsed magnetic field up to $53 \mathrm{~T}$ using a specific setup. Excitonic magnetofingerprints and high-field oscillatory magnetoabsorption yield significant details on the band structure. In addition, the application of an external pressure unveils phenomena that confirm the specific $\mathbf{k} \cdot \mathbf{p}$ model proposed for this compound on the basis of earlier measurements.
\end{abstract}

DOI: 10.1103/PhysRevB.81.205211

PACS number(s): 71.20.Mq, 78.20.Ls, 78.40.Fy

\section{INTRODUCTION}

Indium selenide and other III-VI layered semiconductors have been widely investigated because of their potential applications to fields such as solar energy conversion, ${ }^{1}$ nonlinear optics, ${ }^{2}$ or terahertz generation. ${ }^{3}$ At a more fundamental level, the band structure of InSe and, more specifically, its changes under high pressure, presents some specific features related to the strong anisotropy of its electronic structure and compressibility. ${ }^{4}$ In particular, the emergence of a ringshaped valence-band (VB) maximum predicted by ab initio band-structure calculations ${ }^{5}$ was investigated by photoluminescence and transport measurements under pressure. ${ }^{6,7}$ In fact, high-field magneto-optical measurements under high pressure at low temperature have been recently shown to be a powerful tool for the investigation of the electronic structure of semiconductors and luminescent materials. ${ }^{8-12}$ In this paper we report on an investigation of the peculiar electronic structure of the layered semiconductor InSe by magnetooptical experiments under high pressure up to $5 \mathrm{GPa}$ taking advantage of the recent development of a specific setup allowing to perform magnetoabsorption spectroscopy under pulsed magnetic field up to $60 \mathrm{~T}$ on compressed samples within a diamond-anvil cell (DAC).

Strong magnetic fields are known to quantize the energy states of the charge carriers in semiconductors. This quantization of the electronic states into Landau levels (LLs) and the modification of the electronic wave functions yield the emergence of many different quantum phenomena under high magnetic fields such as cyclotron resonance which corresponds to intraband transitions between LLs either within the conduction or within the valence band. Cyclotron resonance experiments enable to probe, in a quite straightforward way, either the electron or the hole effective mass by intraband low-energy transition with $\Delta N=1$. However, the energy range probed from the bottom of the band is necessarily small and mainly limited by the achievable high magnetic field strength and the scarce infrared laser lines available. Moreover, as the involved midinfrared and far-infrared radia- tion wavelengths $\lambda \sim 10-160 \mu \mathrm{m}$ are similar with sample and pressure chamber typical dimensions in DAC experiments, diffraction can be a serious issue for the realization of such experiments under high pressure. Actually, interband dipolar transitions between orbiting holes and electrons may also occur yielding the appearance of several new absorption bands above the band edge. This effect known as oscillatory magnetoabsorption (OMA) has already been observed in a wide variety of semiconductors such as InAs, Ge and $\mathrm{InSb},{ }^{13,14} \mathrm{GaSe},{ }^{15}$ or InP. ${ }^{16}$ OMA experiments appear as a powerful tool to probe the reduced effective mass perpendicular to the applied field and the band nonparabolicity in a wide energy range above the band edge with optical measurements that can be combined with high-pressure DACs as demonstrated in the present report.

To describe the peculiar electronic and optical properties of the indium selenide within an intuitive framework, a specific $\mathbf{k} \cdot \mathbf{p}$ model has been recently developed for this material, which aims to apply also to the neighbor compounds in the layered III-VI family. ${ }^{5}$ This model takes into account (i) the original layered structure, (ii) the variety of both optical and electrical previous measurements and, in particular, high-pressure studies ${ }^{6,7,17-19}$ as well as (iii) modern $a b$ initio calculations. The most striking feature is the fact that the electron and hole effective masses in the plane of the layers are not governed by the direct gap $E_{g}$ optical matrix element but by higher energy transition $E_{1}^{\prime}$ matrix elements. One of the objective of this paper is to test the validity of this specific $\mathbf{k} \cdot \mathbf{p}$ model. After describing the experimental setup in Sec. II, we will introduce, in Sec. III, the theoretical framework required for the interpretation and discussion of results that are presented in Sec. IV.

\section{EXPERIMENTAL DETAILS}

These experiments have been performed owing to the upgrade of the high-pressure magnetophotoluminescence setup developed at the Laboratoire National des Champs Magnétiques Intenses (LNCMI), the French pulsed magnetic field 
facility at Toulouse. Details on the high-pressure cell, the pulsed magnetic field, and the magneto-optical experiments synchronization can be found elsewhere. ${ }^{8,20}$ To enable magnetoabsorption measurements, a dedicated cell holder and a particular set of optical fiber probes adapted to the specific high-pressure cell have been designed, machined and assembled. This setup allows to feed white light to the sample, with eight $100 \mu \mathrm{m}$ diameter silica fibers embedded in a ceramic needle, through the bottom diamond holder hole, and to collect the light transmitted through the pressure chamber with another bundle of eight identical fibers. The geometry restricts the experiments to the Faraday configuration with $\mathbf{E} \perp \mathbf{B}$. The luminescence of both the sample and/or the ruby sphere pressure gauge can be excited through an appropriate derivation in the collection optical bundle. A supercontinuum fiber laser is used as high power light source over a broad spectral range. The transmitted light is sent either to the entrance slit of a $0.3 \mathrm{~m}$ focal length near infrared spectrometer with a 512 InGaAs photodiode array (sensitive from 750 to $1600 \mathrm{~nm}$ ) having a short $0.5 \mathrm{~ms}$ readout time or to a $0.3 \mathrm{~m}$ visible spectrometer with a $1340 \times 100$ pixels silicon charge coupled device detector (sensitive from 500 to $900 \mathrm{~nm}$ ). A broad spectral range is then available far above the band edge lying around $950 \mathrm{~nm}$ and strongly blueshifted under pressure. We collected numerous spectra during the decay of each field pulse with a few millisecond accumulation time owing to the high luminosity of the white light source. This in turn allows to follow accurately the magnetofingerprints. The samples are $\gamma$-InSe Bridgman grown single crystals cleaved at a thickness of about $25 \mu \mathrm{m}$ to avoid spurious intense interference effects and cut $(200 \mu \mathrm{m} \times 200 \mu \mathrm{m})$ to fit the compression chamber size. A two-step hole, with a $\sim 150 \mu \mathrm{m}$ bottom diameter has been drilled in the nonmagnetic gasket based on Inconel 718 to create the pressure chamber and ensure that all the transmitted light collected has traveled through the crystal (see Fig. 1). The pressure transmitting medium is a 4:1 methanol-ethanol mixture which remains quasihydrostatic in the considered pressure range even at cryogenic temperature. ${ }^{10}$ The pressure is measured in situ at low temperature by the ruby luminescence method, using micrometric ruby spheres, just before and after the magneto-optical measurements at each pressure point. $^{21}$

\section{THEORETICAL FRAMEWORK: EXCITON DIAMAGNETISM AND OSCILLATORY MAGNETOABSORPTION}

In fact, whereas numeric-atomic-orbital-densityfunctional-theory (NAO-DFT) calculations allow to get a precise insight onto the effects of an external pressure on the InSe electronic band structure, such an accurate simulation tool concerning the effect of an external magnetic field is still lacking. Besides, in this compound with strong excitonic character, a major issue for both analytical and numerical simulations arises from the intrinsic spherical symmetry of the Coulomb interaction, whereas the dipolar applied magnetic field is characterized by a cylindrical symmetry. Hence, to get a picture of the electronic structure of such a com-

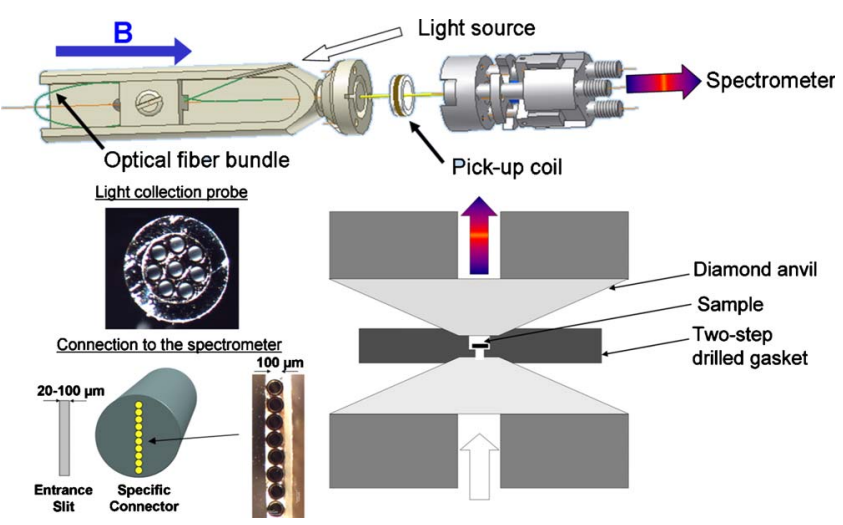

FIG. 1. (Color online) Schematic view of the magnetoabsorption setup, featuring the high-pressure cell and the specific coupling adaptor, allowing the $U$-turn of the optical fibers feeding the white light (white large arrow) to the bottom diamond side of the sample, in the Faraday configuration. A special focus is provided (left) on the aligned fiber connector to enhance the optical coupling with the entrance slit of the spectrometer and (right) on the two-step hole defining the pressure chamber and ensuring that the light driven to the spectrometer (rainbow large arrow) has traveled through the sample.

pound, we will restrict ourselves and present in the following the two usual limits of weak and strong magnetic field. A good scaling parameter for the Coulomb electron-hole interaction is given by the effective Rydberg $R^{*}$, the binding energy of the exciton ground state in a hydrogenlike picture. In InSe, from previous studies we know that $R^{*}=14 \mathrm{meV} .{ }^{24,25}$ On the other hand, a strong magnetic field may quantize electron and hole states into LLs, and a characteristic energy is given by: $\frac{\hbar e B}{2 \mu_{\perp}}=\frac{1}{2} \hbar \widetilde{\omega}_{c \perp}$, where $\widetilde{\omega}_{c \perp}$ is the cyclotron angular frequency. Hence, the ratio $\gamma=\frac{\hbar e B}{2 \mu_{\perp}} / R^{*}$ indicates the magnetic regime. With $\mu_{\perp}=0.117 m_{0}, \gamma=1$ corresponds to $B \approx 30 \mathrm{~T}$. Therefore, going from 0 to $55 \mathrm{~T}$ we can investigate three distinct cases: (i) weak field limit: if $B<20 \mathrm{~T}$, i.e., $\gamma<1$ : the magnetic field effects can be treated as a small perturbation, (ii) strong field limit: $B>40 \mathrm{~T}$, i.e., $\gamma>1$ : the band structure is highly modified by the Landau quantization, and the Coulomb attraction between orbiting electrons and holes can be treated as a small perturbation of the Landau states and (iii) the intermediate region, between 20 and $40 \mathrm{~T}$, i.e., around $\gamma=1$ : the magnetic and electron-hole interaction effects have similar magnitude and the situation is highly nontrivial. Several different theories have been proposed to correlate purely spherical excitonic states to essentially cylindrical high-field wave functions. ${ }^{26-28}$

The low-field perturbation introduced by the magnetic field for an hydrogenlike $s$ excitonic state has already been investigated, in particular, in the case of slightly and strongly anisotropic materials. One can write the diamagnetic shift as ${ }^{15,25,28,29}$

$$
\Delta E_{1 s}=\frac{\hbar^{4}}{32 \pi e^{2}} \frac{\epsilon_{\perp}^{2}}{\mu_{\perp}^{2}} \frac{1}{3}\left(\frac{2}{\epsilon_{\perp} \mu_{\perp}}+\frac{1}{\epsilon_{\|} \mu_{\|}}\right) B^{2}=\sigma_{\|} B^{2},
$$

where $\sigma_{\|}$is the diamagnetic coefficient in electron volt per square tesla when $\mathbf{B} \| \mathbf{c}, \boldsymbol{\epsilon}_{\|, \perp}$ stand, respectively, for the di- 
TABLE I. Admitted values for InSe optical and electrical parameters: static and dynamic dielectric permittivities and electron and hole effective masses along the $\mathbf{c}$ axis and in the layer plane.

\begin{tabular}{lccccc}
\hline \hline & $m_{e}^{*}$ & $m_{h}^{*}$ & $\mu$ & $\epsilon_{\infty}$ & $\epsilon_{0}$ \\
\hline$\| c$ & $0.081 m_{0}$ & $0.17 m_{0}$ & $0.055 m_{0}$ & 7.0 & 7.6 \\
$\perp c$ & $0.138 m_{0}$ & $0.73 m_{0}$ & $0.116 m_{0}$ & 7.34 & 10.3 \\
Methods & Cyclotron resonance & Photoluminescence & & Reflectivity & Reflectivity \\
References & 17 & 19 & & 22 & 23 \\
\hline \hline
\end{tabular}

electric permittivities along $\mathbf{c}$ and in the layer plane and $\mu_{\perp, \|}$ are the reduced effective masses. With the values gathered in Table I, we get $\sigma_{\|}=5.0 \times 10^{-6} \mathrm{eV} / \mathrm{T}^{2}$. One has also to consider the Zeeman splitting which arises from the S.B term and lifts the spin degeneracy of the $s$ state by a factor $\Delta E_{\text {Zeeman }}=m_{S} g \mu_{B} B$.

When the applied magnetic field is very strong, the motion of every charged quasiparticles is severely perturbated and can be well described in term of the so-called quantized LLs. Both the conduction and the valence band give rise to $N$ subbands that in turn affect strongly the observable physical properties of the system. In an electron gas submitted to a magnetic field, the energy states corresponding to the electron motion perpendicular to the magnetic field can be described by an equivalent harmonic oscillator and the eigenvalue spectrum reads

$$
E_{e, h}^{\mathrm{LL}}(N, B)=\left(N+\frac{1}{2}\right) \frac{\hbar e B}{m_{0}}
$$

with $N=0,1,2, \ldots$ Therefore, in the parabolic band approximation, the behavior of Bloch electrons and holes can formally be treated in the same framework, using the effective mass $m_{e, h}^{*}$ instead of the free electron mass $m_{0}$ and one obtains, setting the energy scale zero at the valence-band maximum when $B=0$, the electron LL spectrum as

$$
E_{e}^{\mathrm{LL}}(N, B)=E_{g}+\left(N+\frac{1}{2}\right) \frac{\hbar e B}{m_{e}^{*}}
$$

and the hole LL spectrum, using a positive value for $m_{h}^{*}$ as

$$
E_{h}^{\mathrm{LL}}(N, B)=-\left(N+\frac{1}{2}\right) \frac{\hbar e B}{m_{h}^{*}} .
$$

However, a more realistic description would require to take into account several contributions arising from the deviation to this simple picture as the band nonparabolicity, the valence-band degeneracy, and Coulomb interactions between orbiting electrons and holes.

As each LL is characterized by an angular momentum, which is to be conserved in a dipolar transition, strict selection rules apply on the interband LL OMA transitions. In the Faraday configuration, from the Fermi golden rule we get $\Delta N=N_{\text {Electrons }}-N_{\text {Holes }}=0$ for a direct allowed transition. The possibility of these new OMA transitions under magnetic field strongly modifies the absorption coefficient reflecting the oscillating density of LL states above the band edge. Let us recall that $\alpha$ can be written as

$$
\alpha(\omega)=-\frac{1}{d} \ln \left[\frac{I_{t}(\omega)}{I_{0}(\omega)}\right],
$$

where $I_{t}(\omega)$ is the transmitted light energy density spectrum, $d$ is the sample thickness, and $I_{0}(\omega)$ is the incoming reference energy density spectrum. Above a direct gap and without applied magnetic field the three-dimensional density of states $(\mathrm{DoS}) g(E) \propto \sqrt{E}$ yields an absorption coefficient (neglecting electron-hole interactions for simplicity)

$$
\alpha(B=0, \omega)=\alpha_{0}(\omega) \propto \sqrt{\hbar \omega-E_{g}} .
$$

Under quantizing magnetic field $\alpha$ becomes

$$
\alpha(B, \omega) \propto B \sum_{N} \frac{1}{\sqrt{\hbar \omega-\left[E_{e}^{\mathrm{LL}}(N)-E_{h}^{\mathrm{LL}}(N)\right]}}
$$

and $\alpha$ diverges each time that $\hbar \omega-\left(E_{e}^{\mathrm{LL}}-E_{h}^{\mathrm{LL}}\right)=0$, that is to say each time that the incoming photon energy equals the energy difference between the electron LL and the hole LL defined by the same $N$. These divergences describe the appearance of the OMA bands. Assuming that Eqs. (3) and (4) give the LL energies $E_{e}^{\mathrm{LL}}(N)$ an $E_{h}^{\mathrm{LL}}(N)$, the OMA spectrum $\widetilde{E}(N)$ is given by

$$
\begin{aligned}
\widetilde{E}(N) & =E_{g}+\left(N+\frac{1}{2}\right) \frac{\hbar e B}{m_{e}^{*}}+\left(N+\frac{1}{2}\right) \frac{\hbar e B}{m_{h}^{*}} \\
& =E_{g}+\left(N+\frac{1}{2}\right) \hbar \widetilde{\omega}_{c} \text { with } \widetilde{\omega}_{c}=\frac{e B}{\mu} .
\end{aligned}
$$

We recall that the reduced effective mass $\mu_{\perp, \|}$ is defined as

$$
\frac{1}{\mu_{\perp, \|}}=\frac{1}{m_{e \perp, \|}^{*}}+\frac{1}{m_{h \perp, \|}^{*}} .
$$

As usual, the formal divergences are in fact smeared out by scattering processes reducing the mobility of the charge carriers and broadening the LLs. Assuming a Lorentzian broadening characterized by a phenomenological $\Gamma$ parameter, $\alpha(B, \omega)$ becomes

$$
\alpha(B, \omega) \propto B \sum_{N} \sqrt{\frac{\omega-\omega_{N}+\sqrt{\left(\omega-\omega_{N}\right)^{2}+\Gamma^{2}}}{2 \hbar\left[\left(\omega-\omega_{N}\right)^{2}+\Gamma^{2}\right]}},
$$

where $\widetilde{\omega}_{N}=\widetilde{E}(N) / \hbar \cdot{ }^{30}$ In fact, the physical quantity we actually measure in our experiments corresponds to the normalized absorption coefficient $\widetilde{\alpha}(\omega)=\alpha(B, \omega)-\alpha_{0}(\omega)$, that is, 


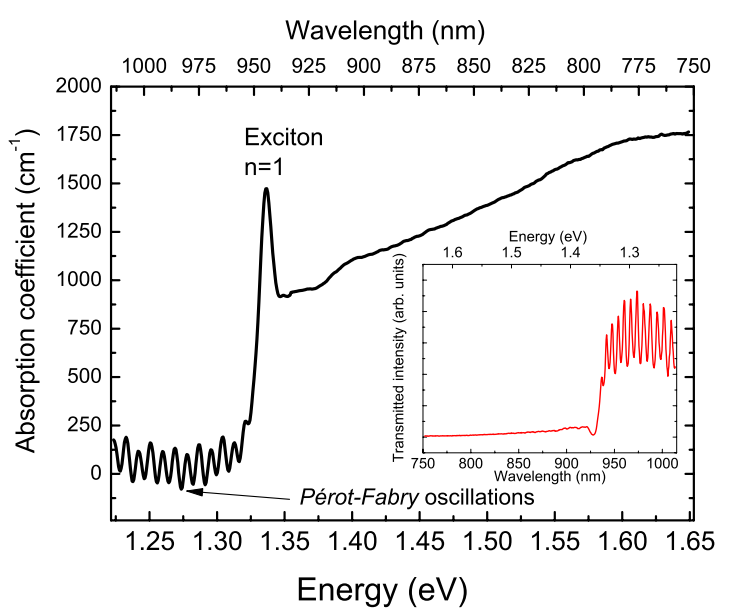

FIG. 2. (Color online) Typical absorption coefficient spectra in the fundamental InSe band-gap region measured in the diamondanvil cell at $4 \mathrm{~K}$. Pérot-Fabry oscillations are clearly noticeable below the gap as well as the $n=1$ excitonic peak. Inset: raw transmitted light data.

$$
\begin{aligned}
\widetilde{\alpha}(\omega) & =-\frac{1}{d} \ln \left[\frac{I_{t}(\omega, B)}{I_{0}(\omega)}\right]+\frac{1}{d} \ln \left[\frac{I_{t}(\omega, B=0)}{I_{0}(\omega)}\right] \\
& =-\frac{1}{d} \ln \left[\frac{I_{t}(\omega, B)}{I_{t}(\omega, B=0)}\right] .
\end{aligned}
$$

\section{RESULTS AND DISCUSSION}

\section{A. Low temperature, high pressure: General trends}

In the Fig. 2 we show the absorption coefficient spectrum measured in the DAC at low temperature $(4 \mathrm{~K})$ and ambient pressure. One can easily distinguish three different phenomena: (i) a direct gap absorption step around $1.40 \mathrm{eV}$, (ii) a sharp peak related to the $n=1$ excitonic state, and (iii) typical Pérot-Fabry oscillations below the band edge $E<E_{g}$. These oscillations arise from the low absorption coefficient $\alpha$ $\sim 10^{3} \mathrm{~cm}^{-1}$, the layered structure of this compound that allows one to cleave it easily and obtain highly reflecting surfaces and the low thickness of the sample $d \sim 20 \mu \mathrm{m}$. The crystal acts as a Pérot-Fabry cavity and is transparent only for a few wavelengths $\lambda(i)$. This provides an accurate measurement of the sample thickness $d$ from a couple of consecutive $\lambda(i)$,

$$
d=\frac{\lambda(i+1) \lambda(i)}{2 n[\lambda(i+1)-\lambda(i)]},
$$

where $n$ is the optical index around $\lambda(i)$. From the spectrum we show in Fig. 2 we get $d=26.2 \mu \mathrm{m}$ using $n=2.71$. Given the gasket configuration (see Fig. 1), the incoming reference energy $I_{0}(\omega)$ can be measured only in the absence of a sample. Hence, the obtained absorption coefficient is only approximated. However, our raw spectrum is very similar to the best spectra already obtained at ambient pressure in Refs. 24 and 31: hence, this indicates that the loading into the DAC did not alter the crystalline quality of the tiny sample.

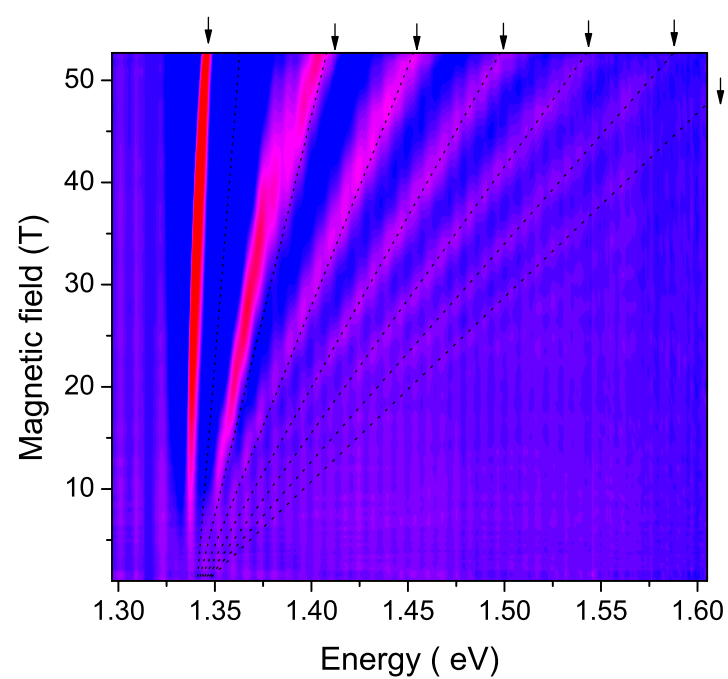

FIG. 3. (Color online) Low-temperature OMA of InSe at ambient pressure up to $53 \mathrm{~T}$ contour map. The normalized absorption intensity is shown in color scale. Excitonic peak around $1.34 \mathrm{eV}$ and OMA bands (arrows) with linear magnetic blueshift are evidenced.

The general trends of the exciton behavior under pressure at zero field are also consistent with previous studies, ${ }^{25,32}$ with a decreasing energy at low pressure followed by a superlinear increase, coupled with a progressive decrease in the intensity leading to the loss of the exciton peak above $\sim 3 \mathrm{GPa}$.

\section{B. Oscillatory magnetoabsorption at ambient pressure}

\section{General trends}

Previous works of InSe magneto-optical properties are scarce and limited to very low field, with a modulationspectroscopy study up to $5 \mathrm{~T}$ (Ref. 33) showing the $n=1, n$ $=2$, and $n=3$ excitonic states and structure attributed to the $N=2$ and $N=3$ LLs. A reduced perpendicular mass $\mu_{\perp}$ $=0.090 \pm 0.010 m_{0}$ is proposed together with a value for $\sigma_{\perp}$ $=14 \pm 2 \times 10^{-6} \mathrm{eV} / \mathrm{T}^{2}$. Another work by magnetoabsorption up to $38 \mathrm{~T}$ (Ref. 34) but focused on the excitonic feature around the $E_{1}^{\prime}$ transition provides a reduced mass $\mu_{\perp}$ $=0.119 \pm 0.007 m_{0}$. Hence, our high-field study up to $53 \mathrm{~T}$ extends largely the explored domain of the phase diagram.

The applied magnetic field strongly affects the optical properties of InSe as it appears in Figs. 3 and 4 where we show a contour map of the absorption in the energy magnetic field plane. Even if the excitonic peak around $1.34 \mathrm{eV}$ is only slightly displaced, one should note easily the onset of the OMA bands, when the magnetic field is stronger than $\sim 20 \mathrm{~T}$. These new bands are blueshifted by the increasing field. As usual ${ }^{13}$ we plot also the normalized spectrum, using the spectrum at zero field as reference: $I_{t}(E, B) / I_{t}(E, B=0)$. In this way the oscillatory behavior of the absorption coefficient is enhanced and we gain some sensibility [compare Figs. 4(a) and 4(b)]. A fair qualitative agreement is found with the simple model previously developed. One can then pick out the energy of each OMA band on the different spec- 

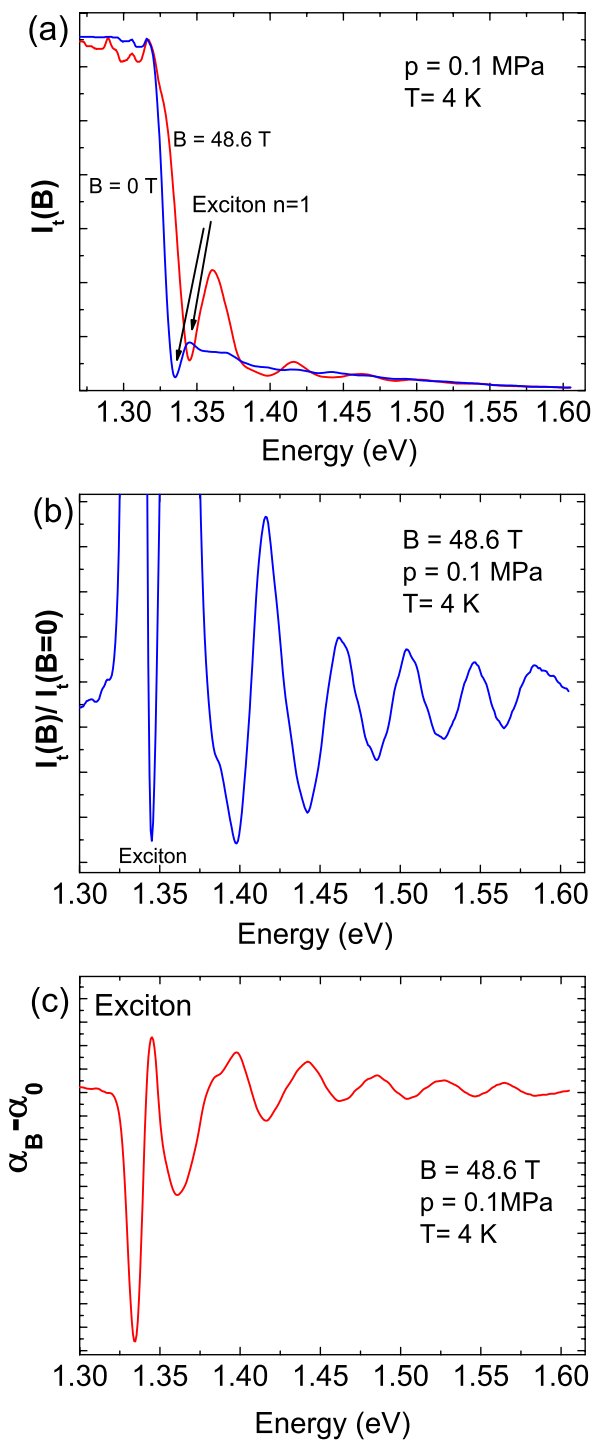

FIG. 4. (Color online) Typical OMA data at $4 \mathrm{~K}$ and $49 \mathrm{~T}$. (a) $I_{t}$ and $I_{t}(E, B=0)$. (b) Normalized intensity $I_{t}(E, B) / I_{t}(E, B=0)$. (c) $\alpha_{B}-\alpha_{0}$.

tra corresponding to different values of the applied magnetic field and plot the OMA fan chart $E(B)$ shown in Fig. 5. This fan chart gives a better insight of the OMA behavior observed in InSe. The Landau quantization is clearly evidenced, with energy shifts linear in $B$, and we can follow up to eight transitions up to $250 \mathrm{meV}$ above the band edge. High-energy transitions with $N>3$ fit particularly well with the linear LL model. Note that in the absence of a polarization control the Zeeman effect cannot be quantified and thereby we measure at the same time the $\sigma^{+}$and $\sigma^{-}$transitions. Using $g \sim 2$ as observed in $\mathrm{GaSe},{ }^{15}$ we expect a Zeeman splitting $g \mu_{B} B \sim 6 \mathrm{meV}$ at $50 \mathrm{~T}$ which cannot be evidenced due to the OMA band width (see Fig. 4).

However, the transitions lying closer to the band edge with $N \leq 2$ exhibit a different behavior, especially below 40 $\mathrm{T}$ that we tentatively assign to Coulomb interactions between orbiting electrons and holes. By the way, the discrepancy is reduced as the field is increased, i.e., when the cyclotron energy over binding-energy ratio $\gamma$ is increased away from

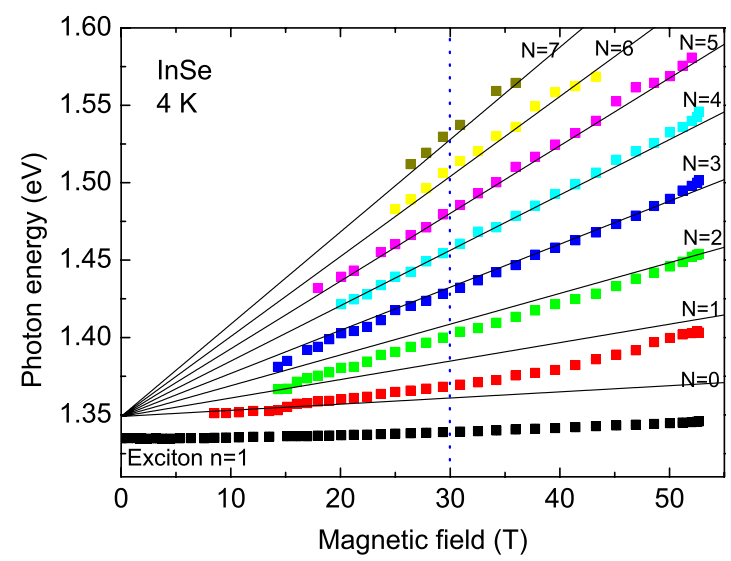

FIG. 5. (Color online) OMA fan chart at ambient pressure and low temperature.

the intermediate region. In fact, there are several papers dealing with magneto-optical properties of the neighbor compound GaSe, partly due to the higher binding energy $R_{\mathrm{GaSe}}^{*}$ $=22.5 \mathrm{meV}$. However, the magnetofingerprints are not really well understood, especially in the low-field region even if the large $N$ transitions are quite consistent with linear LLs. ${ }^{15,35,36}$ One can also notice on the contour map a feature similar with an avoided level crossing on the $N=1$ level around $42 \mathrm{~T}$ $(\sim 1.35 \mathrm{eV})$. In fact, as the energy difference between the linear LL $N=1$ and the observed level crossing is equal to the longitudinal-optical (LO) phonon $26 \mathrm{meV}$ which is known to give rise to an absorption peak at $E_{E x c}+\mathrm{LO}$ (Ref. 37) at zero field and also to magnetopolaron signatures in cyclotron resonance experiments, ${ }^{38}$ this anomaly can probably be attributed to electron-phonon coupling.

\section{Field-induced enhancement of the binding energy}

Figure 6 is focused on the lower energy transition, that is the excitonic ground state at zero field. Together with our data up to $53 \mathrm{~T}$ we present the low-field points from Ref. 33 up to $5 \mathrm{~T}$ and three quadratic $\left(\propto B^{2}\right)$ fits corresponding to (i)

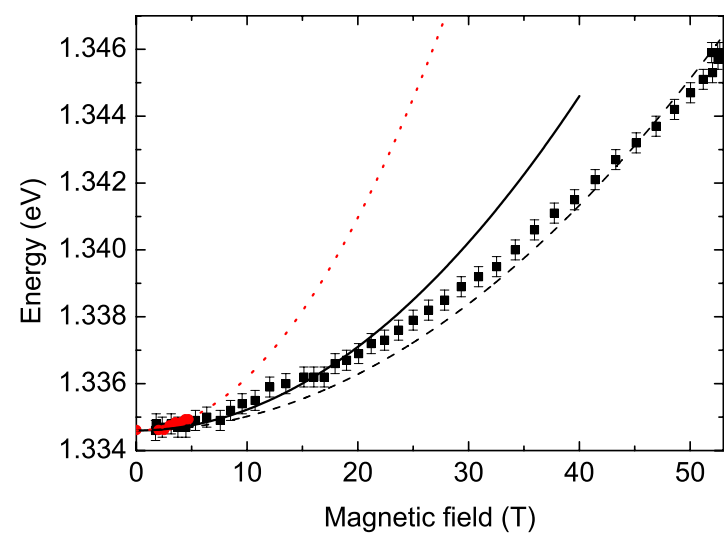

FIG. 6. (Color online) Excitonic peak magnetic behavior. Our data (black squares) and low-field data from Ref. 33 (red dots) are shown as well as three different purely $B^{2}$ fits: in the full magnetic range (dotted black line), in the low-field region $B \leq 20 \mathrm{~T}$ (black solid line) and on low-field data (red dotted line). 
our data below $20 \mathrm{~T}$ in the weak field regime, (ii) low-field data, and (iii) our data in the whole magnetic field explored range. Note first the low value of the total-energy shift $\Delta E_{E x c}=11 \mathrm{meV}$ between 0 and $53 \mathrm{~T}$. The best agreement is found when we restrict the quadratic fit to the weak-field regime, and thus provides a fairly accurate experimental value for the diamagnetic coefficient $\sigma_{\|}=5.7 \times 10^{-6} \mathrm{eV} / \mathrm{T}^{2}$ to compare with the expected value $\sigma_{\|}=5.0 \times 10^{-6} \mathrm{eV} / \mathrm{T}^{2}$ and the previously quoted value $\sigma_{\|}=14 \times 10^{-6} \mathrm{eV} / \mathrm{T}^{2} .^{33}$

In fact, the high-field behavior of this transition evidences a field-induced enhancement of the binding energy. If we assumed a fixed binding energy $R^{*}=14 \mathrm{meV}$ for the exciton, its energy would increase following the gap opening owing to the Landau quantization in the high-field regime,

$$
E_{E x c}=E_{g}-R^{*}(0)+\frac{1}{2} \hbar \widetilde{\omega}_{c}=E_{E x c}(0)+\beta B,
$$

where $\beta=\frac{\hbar e}{2 \mu_{\perp}}$. However, even if the increase is roughly linear above $B>40$ T the extracted value of the reduced effective mass is far from the expected value as we get $\beta_{\text {exp }}$ $=3.1 \times 10^{-4} \mathrm{eV} / \mathrm{T}$, i.e., $\mu_{\text {perp }}=0.180 m_{0}$ whereas we expect $\beta_{1}=4.92 \times 10^{-4} \mathrm{eV} / \mathrm{T}$ from $\mu_{\perp}=0.117 m_{0}$. On the contrary we can estimate the increase in the binding energy under strong magnetic field by

$$
\widetilde{R}^{*}(B)=E_{g}+\beta_{1} B-\widetilde{E}_{E x c}(B),
$$

where $\widetilde{E}_{E x c}(B)$ is the measured transition energy. Hence we get $\widetilde{R}^{*}(B) \approx 29 \mathrm{meV} \approx 2 R^{*}(0)$ at $B=50 \mathrm{~T}$ using $E_{g}+\beta_{1} B$ as the effective magnetic field dependent band gap. This result is in turn in good agreement with theoretical studies dealing with hydrogenlike donor site in the adiabatic approximation to treat separately the motions along the field and in the perpendicular plane with variational method. ${ }^{26,27}$ Actually, in Ref. $26 R^{*}(B)$ is found to reach $\approx 2.1 R^{*}(0)$ at $B=50$ T i.e., $\gamma=1.72$ even if this treatment is strictly valid when $\gamma \rightarrow \infty$. The physical interpretation of this enhancement of the binding energy comes from the progressive squeezing of the excitonic wave function. Equivalently, in a one-particle picture, the strong magnetic field restricts the motion of the electrons and holes in the perpendicular plane to closed orbits: this induces an enhancement of the overlap of the electron and hole wave functions and thereby of the Coulomb interaction.

\section{Nonparabolicity estimation}

In the simple linear LL model, the transition energies are well described by Eq. (8). In fact, assuming such LLs one can renormalize the magnetic field as $\widetilde{B}=(N+1 / 2) B$, collapse all data in one plot $E(\widetilde{B})$ and perform only one linear fit as now the energies of the different transitions $\operatorname{read} E(\widetilde{B})$ $=E_{g}+\frac{\hbar e}{\mu_{1}} \widetilde{B}$. From the plot Fig. 5 and focusing only on the high-field regime above $40 \mathrm{~T}$ we get $\mu_{\text {perp }}=0.138 m_{0}$ which is over evaluated as cyclotron resonance provides a reliable basis for the ambient pressure electronic effective mass $m_{e}^{*}$ $=0.138 m_{0} \cdot{ }^{38}$ In fact, in our case it turns out that the transitions are not equally spaced, and that $\left(E_{N+1}-E_{N}\right)>\left(E_{N+2}\right.$ $\left.-E_{N+1}\right)$ which can be interpreted as a band nonparabolicity

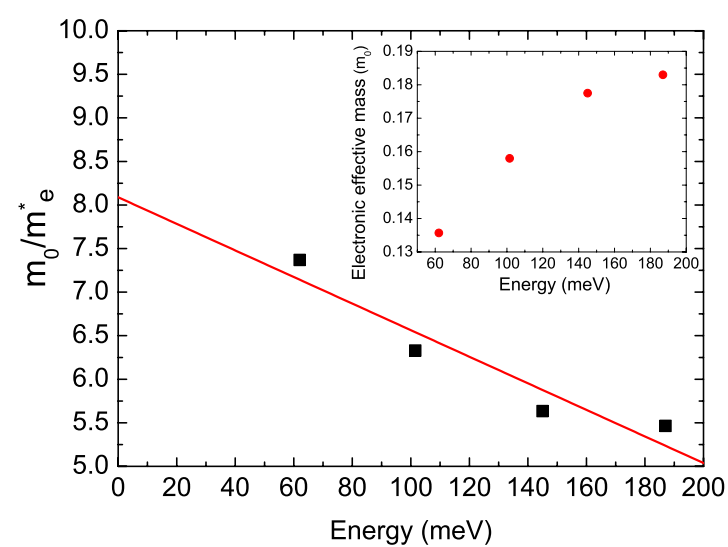

FIG. 7. (Color online) Conduction-band nonparabolicity estimation from OMA values of $1 / m_{e}^{*}(E)$ measured at $52.0 \mathrm{~T}$ plotted as a function of $\widetilde{E}$ and linear fit (solid line).

evidence, i.e., the increase in the effective mass with energy within the conduction and valence bands. As the holes are heavier, the energy range they explore is smaller in our experiments: using $m_{e \perp}^{*}(0)=0.138 m_{0}$ and $m_{h \perp}^{*}(0)=0.73 m_{0}$ we get $\hbar \omega_{c(e)}(0)=42 \mathrm{meV}$ and $\hbar \omega_{c(h)}(0)=8 \mathrm{meV}$ at $50 \mathrm{~T}$. Hence, one can expect stronger influence of the conductionband nonparabolicity on the reduced mass as electrons within the $N=4$ subband lie $\sim 170 \mathrm{meV}$ above the bottom of the band whereas holes in the $N=4$ subbands are only $\sim 30 \mathrm{meV}$ below the valence-band maximum. Therefore, we will assume in the following a parabolic valence band. We now define an energy-dependent reduced effective mass $\mu(N)$, at a fixed value of the magnetic field close to $50 \mathrm{~T}$ as

$$
\mu(N)=\frac{\hbar e B}{\left(E_{N+1}-E_{N}\right)}
$$

and we obtain an energy-dependent electron effective mass,

$$
\frac{1}{m_{e}^{*}(N)}=\frac{\left(E_{N+1}-E_{N}\right)}{\hbar e B}-\frac{1}{m_{h}^{*}(0)} .
$$

The usual formalism to describe nonparabolicity in cyclotron resonance is ${ }^{30}$

$$
\frac{1}{m_{e}^{*}(E)}=\frac{1}{m_{e}^{*}(0)}\left(1+\frac{2 K \tilde{E}}{E_{g}}\right),
$$

where $\widetilde{E}=(N+1) \hbar \omega_{c(e)}(E)$ is the mean energy between the two LLs involved in the considered cyclotron absorption, measured from the bottom of the band. In our framework we get $\widetilde{E}_{N}$ as

$$
\tilde{E}_{N}=\frac{E_{N}+E_{N+1}}{2}-E_{g}-(N+1) \hbar \omega_{c(h)} .
$$

Therefore, using $E_{N}$ values measured at $52.0 \mathrm{~T}$ we can plot $1 / m_{e}^{*}(E)$ as a function of $\widetilde{E}$ in Fig. 7. A linear fit provides then the experimental value for $K=-1.25$ according to Eq. (17) using $E_{g}=1.349 \mathrm{eV}$. The simple model assuming two isolated parabolic band with a $\mathbf{k} \cdot \mathbf{p}$ coupling gives $K=-1$ for a tridimensional compound, but taking also into account the 


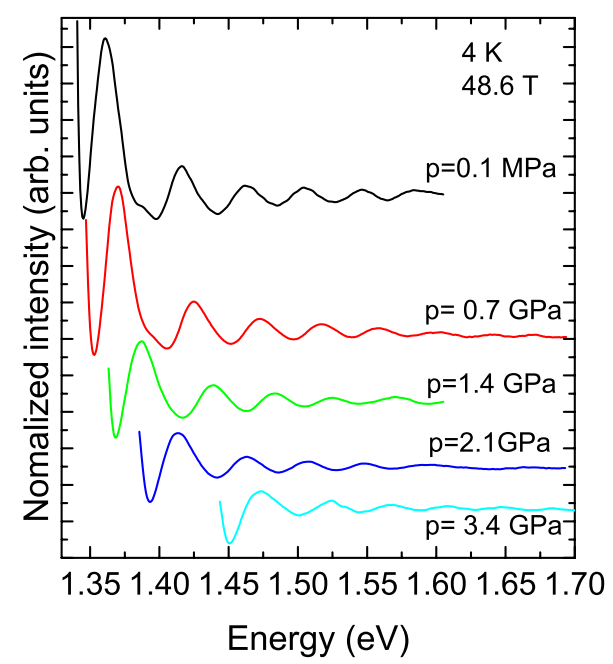

FIG. 8. (Color online) Normalized intensity oscillatory magnetoabsorption spectra at selected pressure.

influence of further bands, $K$ ranges between -0.83 (three bands) (Ref. 39) and -1.5 (five bands, bulk GaAs) (Ref. 40) according to theoretical predictions, and cyclotron resonance studies on GaAs heterostructures give $K=-1.4 \pm 1 .{ }^{41} \mathrm{Be}-$ sides, the effective mass $m_{e}^{*}=0.136$ measured between the lowest transitions is in fair agreement with InSe cyclotron resonance ${ }^{38}$ even if the bottom band extrapolated value $m_{e}^{*}$ $=0.124 m_{0}$ appears to be weak. Using the alternatively proposed hole effective mass $m_{h}^{*}(0)=0.62 m_{0}$ we derive a slightly larger value $m_{e}^{*}=0.128 m_{0}$. Note that Coulomb and spin effect have been neglected in this section. Finally, this measurements provide an original estimation of the conduction-band curvature far from the bottom of the band, in an energy range that cannot be probed by cyclotron resonance due to experimental limitations on the field strength and the existence of a perturbating strong magnetopolaron coupling. ${ }^{42}$

\section{High-field oscillatory magnetoabsorption under high pressure}

As it appears in Fig. 8 the pressure evolution of the OMA fingerprints is smooth. The spectra remain qualitatively similar as the ambient pressure data we show in Fig. 4 with respect to the band edge and exciton energy which are strongly affected by the pressure. The exciton energy pressure dependence is similar with previous works. ${ }^{25,32} \mathrm{We}$ assume in the following that the binding energy decreases with pressure as reported in Ref. 25.

\section{Effective-mass pressure-induced enhancement: An experimental validation for the specific $\mathrm{k} \cdot \mathrm{p}$ model}

We show in Fig. 9 the pressure dependence of the energydependent reduced effective masses $\mu_{\perp}(N)$ we introduced in Sec. IV B 3. A clear increasing tendency is evidenced. Linear fits provide

$$
\begin{aligned}
& \mu_{\perp}(1)=0.121+13 \times 10^{-4} p, \\
& \mu_{\perp}(2)=0.128+11 \times 10^{-4} p,
\end{aligned}
$$

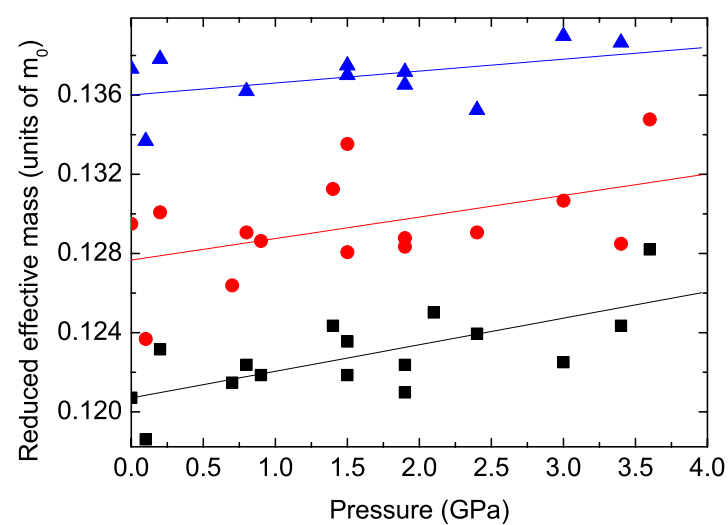

FIG. 9. (Color online) Energy-dependent reduced effective mass $\mu_{\perp}(N)$ pressure behavior: $N=1$ (black squares), $N=2$ (red dots), and $N=3$ (blue triangles).

$$
\mu_{\perp}(3)=0.136+6 \times 10^{-4} p,
$$

where $p$ is in gigapascal, i.e., in terms of relative variation at $4 \mathrm{GPa}$,

$$
\begin{aligned}
& \frac{\Delta \mu_{\perp}(1)}{\mu_{\perp}(1)}(4 \mathrm{GPa})=+4.3 \%, \\
& \frac{\Delta \mu_{\perp}(2)}{\mu_{\perp}(2)}(4 \mathrm{GPa})=+3.5 \%, \\
& \frac{\Delta \mu_{\perp}(3)}{\mu_{\perp}(3)}(4 \mathrm{GPa})=+2 \% .
\end{aligned}
$$

Note that the higher the energy within the band the lower the pressure dependence, even if the data appears somehow scattered partly due to the OMA band asymmetry and large width. Besides, one can compare these relative variations with the pressure-induced evolution of the two main optical transitions $E_{g}$ and $E_{1}^{\prime}$ documented in previous studies, ${ }^{25,32,43}$

$$
\begin{aligned}
& \frac{\Delta E_{g}}{E_{g}}(4 \mathrm{GPa})=13 \%, \\
& \frac{\Delta E_{1}^{\prime}}{E_{1}^{\prime}}(4 \mathrm{GPa})=5 \% .
\end{aligned}
$$

Hence, we demonstrate that the pressure induced increase in the reduced effective mass is not proportional to $E_{g}$ as one could infer from a simple two-bands $\mathbf{k} \cdot \mathbf{p}$ model where the effective mass is simply proportional to the energy gap, but scales under pressure as $E_{1}^{\prime}$. Moreover the pressure behavior of the reduced effective mass does not exhibit any decrease at low pressure as it occurs for the direct gap $E_{g}$ and the mass at $1 \mathrm{GPa}$ is thus heavier than the ambient pressure mass whereas $E_{g}(1 \mathrm{GPa})=E_{g}(0.1 \mathrm{MPa})$. One main assumption of the specific $\mathbf{k} \cdot \mathbf{p}$ model introduced for this compound ${ }^{5}$ is that the effective masses in the layer plane are mainly determined by the $\mathbf{k} \cdot \mathbf{p}$ matrix element describing the coupling between $Z_{1}$ bands and the $Z_{3}$ valence bands at the $Z$ point of the Brillouin zone. Hence, taking into account this peculiarity of InSe the electron effective mass reads 


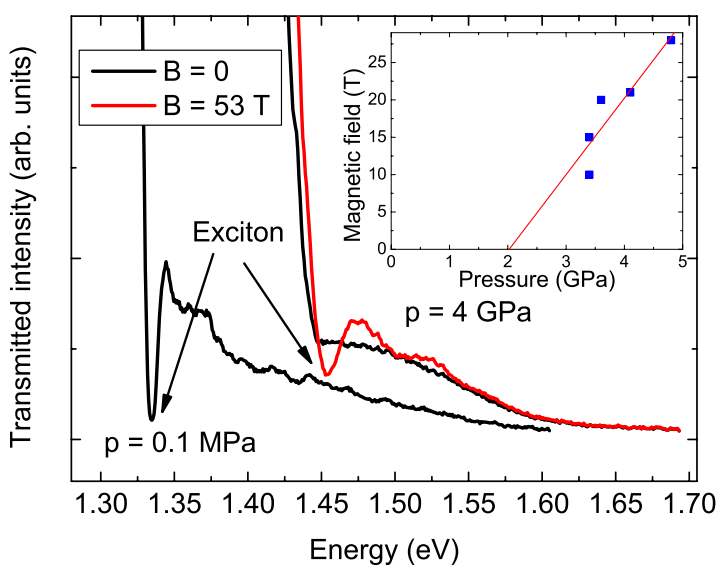

FIG. 10. (Color online) Reentrant exciton behavior at high magnetic field under high pressure. Inset: critical field values $B_{C}$ (squares) as a version of pressure and linear fit (solid line).

$$
\left[\frac{m_{0}}{m_{c Z}^{*}}\right]_{\perp}=1+\frac{2}{m_{0}} \frac{\left|\left\langle c Z_{1}\left|P_{\perp}\right| v Z_{3}\right\rangle\right|^{2}}{E_{c Z 1}-E_{v Z 3}}+\frac{2}{m_{0}} \frac{\left|\left\langle c Z_{1}\left|P_{\perp}\right| c Z_{3}\right\rangle\right|^{2}}{E_{c Z 1}-E_{c Z 3}} .
$$

Recalling that $E_{1}^{\prime}$ correspond to the $E_{v Z 3} \rightarrow E_{c Z 1}$ transitions $5,32,43$ our experimental findings thus represent a striking validation of the specific $\mathbf{k} \cdot \mathbf{p}$ model proposed to describe the unusual band structure of the layered III-VI semiconductors.

\section{Reentrant exciton at high magnetic field under high pressure: A new evidence for a secondary maximum with a lower effective mass}

We now turn to another interesting feature revealed by our high-pressure OMA experiments on this layered material: the reappearance of the exciton absorption peak at high magnetic field under high pressure which constitutes a supplementary evidence for a secondary maximum with a lower effective mass. At zero field, the exciton is no longer observable above $3 \mathrm{GPa}$, as reported earlier by Goñi et al. ${ }^{25}$ However, as it appears in Fig. 10, we recover a strong excitonic absorption peak around 10-15 T. This is consistent with the progressive onset of a secondary maximum for the $Z_{1}$ valence band distant from $Z$ and characterized by a lower effective mass as suggested by previous high-pressure electronic transport measurements and NAO-DFT predictions. ${ }^{7}$ In fact, a very simple model can describe this peculiar exciton reentrant behavior. Assuming that the mass at this ring-shaped secondary maximum $m_{v R}^{*}$ is lighter than the hole effective mass at the $Z$ point $m_{v Z}^{*}$, and using $m_{c Z}^{*}$ to refer to the electron mass at the $Z$ point for clarity, one can describe the Landau quantization band gap opening by

$$
\begin{aligned}
& E_{c Z}\left(B, p_{0}\right)=E_{c Z}\left(B_{0}, p_{0}\right)+\frac{\hbar e B}{2 m_{c Z}^{*}}, \\
& E_{v Z}\left(B, p_{0}\right)=E_{v Z}\left(B_{0}, p_{0}\right)-\frac{\hbar e B}{2 m_{v Z}^{*}},
\end{aligned}
$$

$$
E_{v R}\left(B, p_{0}\right)=E_{v R}\left(B_{0}, p_{0}\right)-\frac{\hbar e B}{2 m_{v R}^{*}} .
$$

At ambient pressure $p_{0}=0.1 \mathrm{MPa}$, the valence-band (VB) maximum in $Z$ is higher in energy than the toroidal-shaped secondary maximum: the exciton is not resonant, has a long lifetime and is clearly distinguishable in the absorption spectrum. Under applied magnetic field, the energy difference $\Delta E_{v}$ between these two $\mathrm{VB}$ maxima reads

$$
\begin{aligned}
\Delta E_{v}\left(p_{0}\right) & =E_{v Z}\left(B, p_{0}\right)-E_{v R}\left(B, p_{0}\right) \\
& =E_{v Z}\left(B_{0}, p_{0}\right)-E_{v R}\left(B_{0}, p_{0}\right)+\frac{\hbar e B}{2}\left(\frac{1}{m_{v R}^{*}}-\frac{1}{m_{v Z}^{*}}\right)
\end{aligned}
$$

and increases with increasing field as $m_{c Z}^{*}>m_{v R}^{*}$. On the other hand, the three extrema are shifted by external pressure, and their behavior can be described using linear pressure coefficients $\alpha, \beta$, and $\delta$ as

$$
\begin{aligned}
& E_{c Z}\left(B_{0}, p\right)=E_{c Z}\left(B_{0}, p_{0}\right)+\alpha p, \\
& E_{v Z}\left(B_{0}, p\right)=E_{v Z}\left(B_{0}, p_{0}\right)+\beta p, \\
& E_{v R}\left(B_{0}, p\right)=E_{v R}\left(B_{0}, p_{0}\right)+\delta p .
\end{aligned}
$$

The differences $(\alpha-\beta)$ and $(\alpha-\delta)$ have already been established through high-pressure absorption experiments and accurate analysis within the Elliot-Toyozawa framework. ${ }^{4}$ As $\delta$ is found to be larger than $\beta$, the secondary maximum energy moves faster than the maximum in $Z$, and a level crossing occurs inducing a direct $\rightarrow$ indirect gap crossover around 2 $\mathrm{GPa}$. Above this pressure $\Delta E_{v}\left(p, B_{0}\right)<0$ the exciton is resonant, its lifetime is strongly reduced and the peak rapidly disappears from the absorption spectrum. Neglecting the pressure-induced effective-mass variation under pressure, and from Eqs. (24) and (25) $\Delta E_{v}(p)$ reads

$$
\begin{aligned}
\Delta E_{v}(p)= & E_{v Z}(B, p)-E_{v R}(B, p) \\
= & E_{v Z}\left(B_{0}, p_{0}\right)-E_{v R}\left(B_{0}, p_{0}\right)-(\delta-\beta) p \\
& +\frac{\hbar e B}{2}\left(\frac{1}{m_{v R}^{*}}-\frac{1}{m_{v Z}^{*}}\right) .
\end{aligned}
$$

Therefore, the positive magnetic contribution to $\Delta E_{v}$ appears to balance the negative pressure contribution and we evidence the existence of a critical magnetic field $B_{C}(p)$ value at each pressure above the direct $\rightarrow$ indirect crossover when $\Delta E_{v}(p)$ becomes positive again, that is to say when the exciton becomes nonresonant again and the exciton peak appears clearly again in the absorption spectrum,

$$
B_{C}(p)=\frac{2(\delta-\beta) p-2\left[E_{v Z}\left(B_{0}, p_{0}\right)-E_{v R}\left(B_{0}, p_{0}\right)\right]}{\hbar e\left(\frac{1}{m_{v R}^{*}}-\frac{1}{m_{v Z}^{*}}\right)} .
$$

Using $(\delta-\beta) \approx 20 \mathrm{meV} / \mathrm{GPa}$ we can obtain and plot $B_{C}(p)$ (see inset of Fig. 10) from which a linear fit gives $B_{C}(p)=$ $-21+10 p$. According to Eq. (27) we then obtain an estimated value for the hole effective at the ring-shaped secondary 
maximum $m_{v R}^{*} \approx 0.03 m_{0}$. In fact, one has to consider that even if this effective mass $m_{v R}^{*}$ is surprisingly low and maybe slightly underestimated, the corresponding DoS (constant at the secondary maximum) would be identical as the DoS at an extremum where the isoenergy surface has a spherical symmetry. Hence, not only the reentrant behavior of the exciton can be interpreted as a signature of a lower effective mass at the secondary maximum, but we claim that the sudden character of this reentrance evidences the peculiarity of the DoS at this secondary maximum. Actually, in the case of a direct $\rightarrow$ indirect crossover with a $\operatorname{DoS} \propto \sqrt{E}$ the number of dispersive final states would increase (decrease) proportionally to the square root of the energy difference between the two maxima. However, in the case of a crossover between the valence-band maximum in $Z$ and the secondary maximum characterized by a toroidal shaped we expect the number of dispersive final states to jump from 0 to a finite constant value (see Ref. 5), which is in fair agreement with the experimental findings. Finally, our OMA experiments thus provide a clear additional evidence for the onset of a toroidal-shaped secondary maximum in the InSe valence band under high pressure.

\section{CONCLUSIONS}

We have performed magnetoabsorption experiments under extreme conditions on the layered semiconductor InSe. These original measurements yield valuable information for the understanding of the peculiar electronic structure of this material. The diamagnetic coefficient and the conductionband nonparabolicity have been documented. Moreover, the pressure-induced modifications of the band structure have been successfully evidenced by the use of magneto-optical spectroscopy. In particular, the scaling of the effective masses with the high-energy $E_{1}^{\prime}$ optical transition has been demonstrated as well as the onset of a secondary maximum for the valence band, characterized by a lower effective mass that we estimate $\approx 0.03 m_{0}$

\section{ACKNOWLEDGMENTS}

Authors acknowledge valuable experimental support by G. Ballon and LNCMI. Part of this work was supported by EuroMagNET II under the EU under Contract No. 228043 at LNCMI-T facility and MALTA-Consolider Ingenio 2010 Program.
*Present address: Department of Earth and Planetary Science, University of California-Berkeley, Berkeley, California 94720, USA.

${ }^{1}$ J. Martínez-Pastor, A. Segura, J. L. Valdés, and A. Chevy, J. Appl. Phys. 62, 1477 (1987).

${ }^{2}$ R. A. Kaindl, F. Eickemeyer, M. Woerner, and T. Elsaesser, Appl. Phys. Lett. 75, 1060 (1999).

${ }^{3}$ W. Shi and Y. Ding, Appl. Phys. Lett. 84, 1635 (2004).

${ }^{4}$ F. J. Manjon, D. Errandonea, A. Segura, V. Munoz, G. Tobias, P. Ordejon, and E. Canadell, Phys. Rev. B 63, 125330 (2001).

${ }^{5}$ A. Segura, F. Manjón, D. Errandonea, J. Pellicer-Porres, V. Muñoz, G. Tobias, P. Ordejón, E. Canadell, A. San Miguel, and D. Sánchez-Portal, Phys. Status Solidi B 235, 267 (2003).

${ }^{6}$ F. J. Manjon, A. Segura, V. Munoz-Sanjose, G. Tobias, P. Ordejon, and E. Canadell, Phys. Rev. B 70, 125201 (2004).

${ }^{7}$ D. Errandonea, A. Segura, F. J. Manjon, A. Chevy, E. Machado, G. Tobias, P. Ordejon, and E. Canadell, Phys. Rev. B 71, 125206 (2005).

${ }^{8}$ M. Millot, J. M. Broto, and J. González, Phys. Rev. B 78, 155125 (2008).

${ }^{9}$ M. Millot, S. Gilliland, J. M. Broto, J. Gonzalez, J. Leotin, A. Chevy, and A. Segura, Phys. Status Solidi B 246, 532 (2009).

${ }^{10}$ M. Millot, J. M. Broto, J. González, and F. Rodriguez, Phys. Rev. B 81, 075120 (2010).

${ }^{11}$ M. Millot, S. George, F. Hatami, W. T. Masselink, J. Leotin, J. González, and J.-M. Broto, High Press. Res. 29, 488 (2009).

${ }^{12}$ R. Valiente, M. Millot, F. Rodríguez, J. González, J. M. Broto, S. George, S. García-Revilla, Y. Romanyuk, and M. Pollnau, High Press. Res. 29, 748 (2009).

${ }^{13}$ S. Zwerdling, B. Lax, L. M. Roth, and K. J. Button, Phys. Rev. 114, 80 (1959).

${ }^{14}$ L. M. Roth, B. Lax, and S. Zwerdling, Phys. Rev. 114, 90 (1959).

${ }^{15}$ K. Watanabe, K. Uchida, and N. Miura, Phys. Rev. B 68,
$155312(2003)$.

${ }^{16}$ P. Rochon and E. Fortin, Phys. Rev. B 12, 5803 (1975).

${ }^{17}$ E. Kress-Rogers, R. J. Nicholas, J. C. Portal, and A. Chevy, Solid State Commun. 44, 379 (1982).

${ }^{18}$ N. Kuroda and Y. Nishina, Physica B \& C 105, 30 (1981).

${ }^{19}$ C. Ferrer-Roca, A. Segura, M. V. Andrés, J. Pellicer, and V. Muñoz, Phys. Rev. B 55, 6981 (1997).

${ }^{20}$ M. Millot, S. George, J.-M. Broto, B. Couzinet, J.-C. Chervin, A. Polian, C. Power, and J. González, High Press. Res. 28, 627 (2008).

${ }^{21}$ K. Nakano, Y. Akahama, Y. Ohishi, and H. Kawamura, Jpn. J. Appl. Phys. 39, 1249 (2000).

${ }^{22}$ F. Manjon, Ph.D. thesis, Universitat de Valencia, 1999.

${ }^{23}$ D. Errandonea, Ph.D. thesis, Universitat de Valencia, 1998.

${ }^{24}$ J. Camassel, P. Merle, H. Mathieu, and A. Chevy, Phys. Rev. B 17, 4718 (1978).

${ }^{25}$ A. R. Goñi, A. Cantarero, U. Schwarz, K. Syassen, and A. Chevy, Phys. Rev. B 45, 4221 (1992).

${ }^{26}$ Y. Yafet, R. W. Keyes, and E. N. Adams, J. Phys. Chem. Solids 1, 137 (1956).

${ }^{27}$ Y. Zhang, A. Mascarenhas, and E. D. Jones, J. Appl. Phys. 83, 448 (1998).

${ }^{28}$ S. Taguchi, T. Goto, M. Takeda, and G. Kido, J. Phys. Soc. Jpn. 57, 3256 (1988)

${ }^{29}$ R. Le Toullec, N. Piccioli, and J. C. Chervin, Phys. Rev. B 22, 6162 (1980).

${ }^{30}$ N. Miura, Physics of Semiconductors in High Magnetic Fields, Semiconductor Science and Technology Vol. 15 (Oxford University Press, New York, 2008).

${ }^{31}$ A. Segura, J. Bouvier, M. V. Andrés, F. J. Manjón, and V. Muñoz, Phys. Rev. B 56, 4075 (1997).

${ }^{32}$ C. Ulrich, M. Moginski, A. Goni, A. Cantarero, U. Schwarz, V. Munoz, and K. Syassen, High Pressure Science and Technology, 
proceedings of the Joint XV AIRAPT and XXXIII EHPRG International Conference, Warsaw, Poland. Sept. 11-15, 1995, edited by W. Trzeciakowski (World Scientific, Singapore, 1996).

${ }^{33}$ G. Saintonge and J. L. Brebner, Phys. Rev. B 30, 1957 (1984).

${ }^{34}$ N. Kuroda, Y. Nishina, H. Hori, and M. Date, J. Phys. Soc. Jpn. 47, 1373 (1979).

${ }^{35}$ A. Rasulov, P. Seiisyan, M. Stam, A. Toropov, and A. Éfros, Sov. Phys. Solid State 29, 3308 (1987).

${ }^{36}$ C. H. Aldrich, C. M. Fowler, R. S. Caird, W. B. Garn, and W. G. Witteman, Phys. Rev. B 23, 3970 (1981).

${ }^{37}$ N. Piccioli, R. Le Toullec, F. Bertrand, and J. C. Chervin, J. Phys. (Paris) 42, 1129 (1981).
${ }^{38}$ R. J. Nicholas et al., Phys. Rev. B 45, 12144 (1992).

${ }^{39}$ E. D. Palik, G. S. Picus, S. Teitler, and R. F. Wallis, Phys. Rev. 122, 475 (1961).

${ }^{40}$ W. Zawadzki, P. Pfeffer, and H. Sigg, Solid State Commun. 53, 777 (1985).

${ }^{41}$ M. A. Hopkins, R. J. Nicholas, M. A. Brummell, J. J. Harris, and C. T. Foxon, Phys. Rev. B 36, 4789 (1987).

${ }^{42}$ D. E. N. Brancus, G. Stan, and A. Dafinei, J. Phys.: Condens. Matter 17, 3241 (2005).

${ }^{43}$ N. Kuroda, O. Ueno, and Y. Nishina, J. Phys. Soc. Jpn. 55, 581 (1986). 